\title{
DE LOS CURSOS DE PUERICULTURA A LA EDUCACIÓN MATERNAL: EL PAPEL DE LAS MATRONAS EN LA PREPARACIÓN PARA EL NACIMIENTO EN ESPAÑA A LO LARGO DEL SIGLO XX*
}

\author{
From childcare courses to maternal education: the role of midwifes \\ in birth preparation in Spain throughout the $20^{\text {th }}$ century
}

\section{Dolores Ruiz-Berdún ${ }^{\alpha}$}

Fecha de recepción: 05/04/2021 • Fecha de aceptación: 03/09/2021

Resumen. Uno de los factores que se ha considerado históricamente más importantes para luchar contra la mortalidad infantil ha sido la educación de las madres. Entre las estrategias utilizadas para mejorarla se encuentra la educación grupal, que hoy es una competencia casi exclusiva de las matronas. Pero no siempre fue así. Este artículo está centrado en el análisis del papel de estas profesionales en la educación de las embarazadas en España, que comenzó con los cursos para madres ofertados por la Escuela Nacional de Puericultura. En 1955 empezaron a popularizarse los cursos para embarazadas destinados a difundir los métodos para el control del dolor en el parto. Los dos primeros libros publicados en España sobre el tema vieron la luz de la mano de una matrona y un médico respectivamente, formados en el extranjero en dichas técnicas. Inicialmente fueron los médicos los más interesados en la difusión, entre las embarazadas, de estas técnicas del control del dolor, llegando a crearse unidades específicas en las grandes residencias sanitarias del tardofranquismo. Algunos ayuntamientos también incorporaron la «preparación al parto» en su cartera

\footnotetext{
* Este artículo se enmarca en el proyecto «De la propaganda sanitaria a la educación para la salud. Ideología, discursos y saberes en la España de Franco (1939-1975)» HAR2015-64150-C2-1-P [MINECO/FEDER, UE]. Mi agradecimiento a las matronas informantes Rosario Muñoz, Teresa Gómez y Mercedes Serrano y en especial a Hortensia García Pacheco. Dedicado a la memoria de Consuelo Ruiz Vélez-Frías.

${ }^{\alpha}$ Departamento de Cirugía, Ciencias Médicas y Sociales, Facultad de Medicina y Ciencias de la Salud, Universidad de Alcalá. Campus Científico-Tecnológico, 28805 Alcalá de Henares (Madrid) España. lola.ruizberdun@uah.es (D) https://orcid.org/0000-0001-8884-6139.
}

Cómo citar este artículo: Ruiz-Berdún, Dolores. «De los cursos de puericultura a la educación maternal: el papel de las matronas en la preparación para el nacimiento en España a lo largo del siglo XX». Historia y Memoria de la Educación 15 (2022): 63-98 
de servicios. Pero la auténtica generalización de la educación maternal llegó de la mano de la incorporación de las matronas a los Centros de Atención Primaria de Salud a finales de los años ochenta.

Palabras clave: Historia de las matronas; Parto sin dolor; Educación maternal; Preparación al parto; Cursos de puericultura.

Abstract. One of the factors that has historically been considered most important in combating child mortality has been the education of mothers. Among the strategies used to improve it is group education, which today is almost exclusively the responsibility of midwives. But this was not always the case. This article focuses on the role of these professionals in the education of pregnant women in Spain, which began with the courses for mothers offered by the Escuela Nacional de Puericultura. From 1955 onwards, courses for pregnant women, aimed at disseminating methods for pain control in childbirth, began to become popular. The first two books published in Spain on the subject were launched in 1955 by a midwife and a doctor respectively, who had had to go abroad to be trained in these techniques. Initially it was the doctors who were most interested in spreading these pain control techniques, and specific units were set up in the large nursing homes of late Francoism. Some town councils also incorporated "birth preparation" into their service portfolio. But the real generalisation of maternal education came with the incorporation of midwives into Primary Health Care Centres at the end of the 1980s.

Keywords: History of Midwives; Painless childbirth; Maternity and birth preparation; Childcare courses.

\section{INTRODUCCIÓN}

Hasta fechas relativamente recientes, la preocupación por la educación de las mujeres en España ha estado más ligada a los beneficios que la «cultura materna» pudiera tener para su futura descendencia que en situar al colectivo femenino en una posición de igualdad respecto a los hombres. ${ }^{1}$ El interés por la educación de embarazadas y madres recientes empezó a cobrar importancia al mismo tiempo que lo hacía el valor social de la infancia, a finales del siglo XIX. ${ }^{2}$ Es en esta época en la que se

\footnotetext{
1 Pilar Ballarín Domingo, «La educación de la mujer española en el siglo xIX», Historia de la Educación. Revista interuniversitaria 8 (1989): 255. Teresa González Pérez, «El aprendizaje de la maternidad: discursos para la educación de las mujeres en España», Convergencia, 46 (2008): 103.

2 Sobre el cambio de mentalidad que se produjo, a nivel internacional, en la consideración social de la infancia existe una abundante bibliografía. Véase, por ejemplo, la monografía Salvad al niño y, concretamente, su introducción donde aparece una amplia revisión de las investigaciones previas
} 
publica una extensa obra en tres tomos titulada precisamente La educación de la mujer, cuya edición se justifica en la siguiente sentencia: «La salvación, la civilización, la felicidad del hombre, de la sociedad, está indefectiblemente en la educación de la mujer». ${ }^{3}$ En esta obra y en otras de la misma temática, la misión de las mujeres en la vida estaba indisolublemente ligada a la maternidad.

La historia de la educación de las madres y futuras madres en España ha sido objeto de investigación bajo distintos puntos de vista. Irene Palacio Lis ha estudiado el adoctrinamiento hacia la maternidad que sufrieron las madres españolas durante el siglo XIx y primera mitad del siglo xx. ${ }^{4}$ Del desarrollo de la nueva ciencia de la Maternología a principios del siglo xx y su divulgación a través de diversos medios, se han ocupado autores como Carmen Colmenar Orzaes, ${ }^{5}$ Modesta Salazar, Josep Bernabeu, ${ }^{6}$ o Isabel Jiménez Lucena et al. ${ }^{7}$ Teresa González Pérez ha analizado la escuela como centro de aprendizaje de la maternidad para las niñas dentro de la estrategia pronatalista del régimen franquista. ${ }^{8}$

Pero si nos centramos exclusivamente en la historia de la educación grupal desde el ámbito sanitario en España, el número de trabajos se

relacionadas con la salud infantil: Enrique Perdiguero Gil y Mercedes Del Cura, «Introducción», en Salvad al niño, comp. Enrique Perdiguero Gil (Valencia: Seminari de Estudis sobre Ciència, 2004), 15-25, y Esteban Rodríguez Ocaña, «La construcción de la salud infantil. Ciencia, medicina y educación en España», Historia Contemporánea, 18 (1998): 19-52.

3 José Panadés y Poblet, La educación de la mujer. Según los más ilustres naturalistas é higienistas de ambos sexos, Tomo I (Barcelona: Jaime Seix y Compañía, 1878), XI.

${ }^{4}$ Véase, por ejemplo, Irene Palacio Lis, “"Consejos a las madres”: autoridad, ciencia e ideología en la construcción social de la función materna. Una mirada al pasado», Sarmiento, n. ${ }^{\circ}$ (2003): 61-79; Irene Palacio Lis, Mujeres ignorantes: madres culpables. Adoctrinamiento y divulgación materno-infantil en la primera mitad del siglo xx (València: Universitar de València, 2003).

${ }_{5}^{5}$ Carmen Colmenar Orzaes, «La institucionalización de la maternología en España durante la Segunda República y el franquismo», Historia de la Educación, n. 28 (2009): 161-183.

6 Modesta Salazar Agulló et al., «Madres y enfermeras: el modelo de cuidados a la infancia en la colección "Al servicio de España y del niño español" (1938-1964)», Recien. Revista científica de Enfermería 1 (2010). http://hdl.handle.net/10045/20315

${ }^{7}$ Isabel Jiménez Lucena, María José Ruiz Somavilla y Jesús Castellanos Guerrero, «Un discurso sanitario para un proyecto político. La educación sanitaria en los medios de comunicación de masas durante el primer franquismo", Asclepio 54, n. ${ }^{\circ} 1$ (2002): 201-218.

8 Véase, por ejemplo: Teresa González Pérez, «El aprendizaje de la maternidad: discursos para la educación de las mujeres en España», Convergencia, n. 46 (2008): 103, y Teresa González Pérez, «Los programas escolares y la transmisión de roles en el franquismo: la educación para la maternidad», Bordón 61, n. ${ }^{\circ} 3$ (2009): 93-105. 
reduce mucho. Algunas tesis centradas en la educación maternal actual hacen un breve repaso histórico, en ocasiones plagado de errores, tal vez, precisamente, por la falta de estudios previos. Por este motivo solo destacaremos la aportación de Matilde Fernández y Fernández-Arroyo en su tesis y en un artículo posterior donde analiza las distintas escuelas de psicoprofilaxis. ${ }^{9}$

La aportación más relevante a la historia de la educación maternal en España ha sido la realizada por Amaya García Arregui en su tesis doctoral Psicoprofilaxis y educación maternal. Historia de la preparación al parto en España. ${ }^{10}$ Dicha tesis viene a llenar un importante vacío historiográfico sobre el tema. Sin embargo, y probablemente debido a la selección de las fuentes, deja muy desdibujado el papel que tuvieron las matronas en dicha historia. ${ }^{11}$ Un ejemplo más de la invisibilización que ha tenido este colectivo profesional dentro de la historia de la medicina hasta épocas recientes.

El objetivo de este trabajo es, precisamente, estudiar la evolución de la educación grupal a las embarazadas y madres recientes en España poniendo el foco en el papel que han tenido las matronas en dicho desarrollo.

Para su realización se han utilizado una gran variedad de fuentes. Uno de los recursos de donde se ha obtenido más información ha sido la prensa profesional relacionada con las matronas durante el franquismo. Revistas como Matronas o Súrgere, que se conservan en la Biblioteca Nacional (BNE) han sido imprescindibles. Otras fuentes primarias hemerógraficas han sido las provenientes de la prensa general (a través de

\footnotetext{
9 Matilde Fernández y Fernández Arroyo, «Elaboración, validación y aplicación de una herramienta de evaluación para los programas de educación maternal, de los centros de Atención Primaria de la Comunidad de Madrid» (Tesis doctoral, Universidad Pontificia de Comillas de Madrid, 2012), 7190 y Matilde Fernández y Fernández-Arroyo, «Análisis comparativo de las principales Escuelas de Educación Maternal», Index de Enfermería 22, n. 1-2 (2013): 40-44.

10 Amaya García Arregui, «Psicoprofilaxis y educación maternal. Historia de la preparación al parto en España» (Tesis doctoral, Universidad Autónoma de Barcelona, 2019).

${ }_{11}$ Recordemos que, en la actualidad, las matronas son, casi exclusivamente, las responsables de los programas educativos para embarazadas que se llevan a cabo dentro del sistema sanitario público. La variabilidad que existe actualmente en los contenidos y la forma de impartir educación grupal a las futuras madres y sus parejas, desde los centros de salud, es enorme y depende de diversos factores, como la ratio de matronas por mujeres en edad fértil, endémicamente exiguo en España, comparado con la mayoría de los países europeos, o las infraestructuras con las que se cuenta.
} 
la Hemeroteca de la Biblioteca Nacional y la hemeroteca del diario $L a$ Vanguardia). También en la BNE se han consultado algunas fuentes primarias bibliográficas. Se ha recurrido a fuentes archivísticas para concretar ciertos aspectos más concretos de la investigación en el Archivo General de la Administración (AGA), el archivo del Instituto de Gestión Sanitaria (INGESA) y el Centro Documental de la Memoria Histórica. Otros materiales impresos, como monografías y libros de actas y documentación de diversos congresos de matronas han sido adquiridos a través de la compra en páginas de coleccionismo. Por último, también han sido muy relevantes las entrevistas realizadas a dos matronas informantes, muy involucradas en el tema de estudio, una de las cuales también aportó fuentes personales para concretar algunos datos.

\section{ANTECEDENTES DE LA EDUCACIÓN PARA MADRES EN ESPAÑA}

Como ya se ha comentado en la introducción, a principios del siglo xx se consolidó el discurso médico que responsabilizaba a las madres de la buena o mala salud de su progenie. ${ }^{12}$ Discurso que determinaba que las preocupantes cifras de morbimortalidad infantil eran la consecuencia de una falta generalizada de "cultura materna» de las españolas, sin tener en cuenta otros factores como la procedencia social o económica de las familias. Preocupación nada nueva si hacemos caso de las palabras del catedrático de partos y enfermedades de mujeres y niños del Real Colegio de Cirugía de San Carlos en 1797:

Lastimado de la excesiva mortandad de los niños he puesto el mayor esmero en averiguar su origen, y he descubierto no ser otro que el mal modo con que generalmente se les cuida desde su nacimiento. ${ }^{13}$

Precisamente los libros de vulgarización sanitaria, como el escrito por Agustín Ginesta, fueron los recursos más utilizados, inicialmente, para

\footnotetext{
12 Sobre la culpabilización de las madres en las cifras de mortalidad infantil véase Palacio Lis:

"“Consejos a las madres" y Palacio Lis, Mujeres ignorantes: madres culpables.

13 Agustín Ginesta, El conservador de los niños (Madrid: En la Imprenta Real, 1797), s.p. No obstante, hay que señalar que hasta principios del siglo xx no se contó, en España, con unas estadísticas fiables con las que poder evaluar la magnitud del problema.
} 
paliar esa supuesta falta de conocimientos maternos. ${ }^{14}$ Durante el siglo XIX se consolidó esta estrategia para formar a las futuras y recientes madres, incorporando estos conceptos en libros de puericultura o de higiene del embarazo. ${ }^{15}$ Pero no hay que olvidar que el analfabetismo femenino, especialmente entre las capas populares de la sociedad, era muy elevado $\mathrm{y}$, por tanto, solo un pequeño grupo de mujeres, probablemente las que menos los necesitaban, podían beneficiarse de este tipo de recursos. ${ }^{16}$

A principios del siglo xx, comenzaron a usarse conceptos como el de «puericultura intrauterina» o "puericultura prenatal», muy relacionados con la corriente eugénica. La necesidad de fomentar el cuidado pre y postnatal era un tema recurrente en las ponencias presentadas en diversas reuniones científicas nacionales, como la que defendió el ginecólogo Adolfo Martínez Cerecedo en el V Congreso para el Progreso de las Ciencias, celebrado en Valladolid en octubre de 1915:

[...] el Gobierno tiene el deber de inspeccional (sic) y corregir las causas de las excesivas defunciones creando el Cuerpo del Registro civil en todas las poblaciones que sea posible, para que lleven un registro de los abortos, partos prematuros y fetos muertos que ocurran, estudiando la etiología y las circunstancias morales, sociales e intelectuales de las familias en que existan tales casos, con el fin de aplicar la profilaxis correspondiente. ${ }^{17}$

En el contexto internacional existía la misma preocupación por las cifras de mortalidad infantil y la educación de las madres. Durante la celebración del Tercer Congreso Internacional de Protección al Niño de

\footnotetext{
${ }_{14}$ Unos años antes, en la primera parte de su Discurso sobre la educación física y moral de las mujeres, Josefa Amar y Borbón incluía este tipo de contenidos destinados a educar a las mujeres en nociones de salud: Josepha Amar y Borbón, Discurso sobre la educación física y moral de las mujeres (Madrid: En la Imprenta de D. Benito Cano,1790). La obra iba dirigida a un público culto y, en todo caso, a mujeres de la burguesía: Antonio Viñao, «La educación en las obras de Josefa Amar y Borbón», Sarmiento, n. ${ }^{\circ} 7$ (2003): 45-47.

15 Sobre la literatura destinada a las madres puede consultarse Esteban Rodríguez Ocaña, «La construcción de la salud infantil. Ciencia, medicina y educación en España», Historia Contemporánea, n. 18 (1998): 19-52 y también Esteban Rodríguez Ocaña y Enrique Perdiguero «Ciencia y persuasión social en la medicalización de la infancia en España, siglos XIX-XX». História, Ciências, Saúde-Manguinhos13, n. ${ }^{\circ}$ 2, (2006): 303-324. doi: https://doi.org/10.1590/S0104-59702006000200007

16 Ballarín Domingo, «La educación de la mujer», 249.

17 Dr. Martínez Cerecedo, «El abandono de la Puericultura Intrauterina en España», España Médica, 5, n. ${ }^{\circ} 180$ (1916): 1.
} 
Pecho, que tuvo lugar en Berlín del 1 al 18 de septiembre de 1911,18 se identificaba a las matronas como un grupo profesional clave en la educación de las madres:

Importa también instruir á las matronas. Son las que están más en contacto con las madres en muchas localidades pequeñas y las que pueden dirigir la lactancia con éxito. Pide con este objeto el Dr. Rissmann, de Osnabrück, que se funden en las escuelas de matronas cursos especiales de puericultura, donde las alumnas adquieran la técnica de la alimentación de un niño sano, para que puedan aconsejar y dirigir la lactancia ó la alimentación artificial, al menos, durante el primer año. ${ }^{19}$

En España, en esos momentos, no existían escuelas de matronas. Tras el cierre de los Reales Colegios de Cirugía, y desde 1845, estas profesionales se formaban en las universidades que contaban con Facultad de Medicina y no tenemos constancia de que se creasen, atendiendo a esta demanda, cursillos específicos de puericultura dirigidos a ellas. El proyecto de construcción de un hospital de mujeres en Madrid en el que se crearía una escuela de matronas estaba paralizado. ${ }^{20}$ Sin embargo, es posible que la asistencia de algunos médicos al congreso de Berlín fuese el impulso definitivo de un proyecto que vería la luz años más tarde: la creación de la Escuela Nacional de Puericultura (ENP). ${ }^{21}$

La Escuela, fundada en Madrid en 1923 por Enrique Suñer, uno de los médicos que había asistido al III Congreso de Niños de Pecho, fue

\footnotetext{
18 En España, la Pediatría seguía ligada a la Ginecología y la Obstetricia, como lo demuestra el hecho de que se celebrase, ese mismo año, un Congreso de Obstetricia, Ginecología y Pediatría: Fernando Ponte Hernando et al., «Tal como éramos. En el centenario del primer Congreso Español de Pediatría de Palma de Mallorca (1914-2014)», Anales de Pediatría 80, n. 6, doi: 10.1016/j.anpedi.2014.03.010

${ }^{19}$ Isidoro de la Villa, «Desde Berlín. El tercer congreso de protección al niño de pecho», España Médica, 1, n. ${ }^{\circ} 25$ (1911): 5.

${ }^{20}$ La impulsora del proyecto de construcción de un hospital para mujeres en el que también se formasen las matronas fue la reina regente María Cristina de Habsburgo-Lorena. Aunque la primera piedra del edificio se puso en 1904, hasta 1924 no se inauguró el centro: Rosario Martín-Alcaide y Dolores Ruiz-Berdún, «Un hospital para mujeres promovido por mujeres: la Casa de Salud de Santa Cristina de Madrid», Temperamentvm, 15 (2019): 1-6. http://ciberindex.com/index.php/t/article/view/ e12642

${ }^{21}$ Ya en 1909 Manuel Tolosa Latour propuso la creación en España de un «Instituto Nacional de Maternología»: Carmen Colmenar Orzaes, «La institucionalización de la maternología».
} 
el primer centro destinado a la especialización médica en nuestro país. Inicialmente adscrita al Consejo Superior de Protección a la Infancia, comenzó con los cursos de formación en 1926 y en ella se formaron «más de 2.000 médicos puericultores y más de 10.000 puericultoras, entre los grados de maestras matronas, enfermeras y diplomadas [...] El primero de los puericultores formados en esta Escuela fue el doctor Juan Bosch Marín»:22

Elevar el nivel cultural sanitario del país y en especial de la mujer; luchar contra los errores de la sabiduría popular, educación sanitaria, cambio de comportamiento, revisión sanitaria sistemática del niño pequeño, hacer posible una mejor higiene escolar, he ahí una serie de objetivos, cumplidos algunos de los cuales han hecho el milagro sanitario que supone que en el año 1900 más de 100.000 niños menores de un año murieran (índice de mortalidad infantil $=186$ por 1.000$)$; bajando en 1935 a 112; 1940 retroceso a 140 (trastornos de la guerra civil y revolución), y llegando por fortuna en 1975 a la tasa muy razonable y halagüeña de 17 por $1.000 .{ }^{23}$

La Escuela contaba con una sección de Puericultura Intrauterina donde, desde su creación, se formaban las matronas e impartían cursos a las madres. Según las estadísticas de la ENP, tan solo se titularon en la institución 171 matronas entre 1926 y 1977, con una distribución irregular a lo largo de los años y desapareciendo totalmente a partir de 1963. Debido a los cambios que experimentó la profesión en los años previos, es posible que su desaparición de las estadísticas se deba a que las matronas pasasen a engrosar la de las enfermeras formadas en la institución,

\footnotetext{
${ }_{22}$ Luciano de la Villa Rodríguez, «Presentación», en Escuela Nacional de Puericultura 1926-1976. Cincuenta aniversario de la actividad docente y asistencial. (Madrid: Ministerio de Sanidad y Seguridad Social, Subsecretaría de la Salud, 1978), 2.

${ }_{23}$ Juan Bosch Marín, «Memoria de la Escuela Nacional de Puericultura e Higiene Infantil. Datos para historia de la puericultura española», en Escuela Nacional de Puericultura 1926-1976. Cincuenta aniversario de la actividad docente y asistencial. (Madrid: Ministerio de Sanidad y Seguridad Social, Subsecretaría de la Salud, 1978), 75. Para saber más sobre la Escuela Nacional de Puericultura puede consultarse: Esteban Rodríguez Ocaña, «Aspectos sociales de la pediatría española anteriores a la Guerra Civil (1936-39)», en La ciencia moderna y el conocimiento del Nuevo Mundo: actas de la I Reunión de Historia de la Ciencia y de la Técnica de los Países Ibéricos e Iberoamericanos, coord. José Luis Peset Reig (Madrid: Consejo Superior de Investigaciones Científicas, 1985), 454.
} 
que alcanzó la cifra de $758 .{ }^{24}$ Probablemente, muchas más matronas se formaron en las diferentes escuelas provinciales de puericultura que se fueron inaugurando a lo largo de todo el territorio nacional.

\section{LA EDUCACIÓN DE LAS MADRES DURANTE LA DICTADURA FRANQUISTA}

Para la dictadura franquista, especialmente durante el periodo autárquico, disminuir la tasa de mortalidad infantil se convirtió en un asunto prioritario. ${ }^{25}$ La enseñanza de la puericultura a las madres se reguló por una orden promulgada a finales de $1941 .{ }^{26}$ La supuesta responsabilidad materna, en las cifras de mortalidad infantil nacionales, quedaba patente en la justificación de la orden: «Para que la ignorancia no constituya causa de mortalidad infantil». La novedad que se añadía, a la ya instituida educación sanitaria individual, era la organización de cursillos colectivos mensuales obligatorios.

Esta obligatoriedad impuesta era la consecuencia, según los sanitarios, del poco interés mostrado por las usuarias en acudir a los centros maternales: «Las mujeres ignoran cómo tienen que prepararse para ser madres y no hay que decir que no solo desconocen, sino que no quieren aprender a criar a sus hijos $[\ldots] » .{ }^{27}$

El lugar para impartir las enseñanzas serían los Dispensarios de Puericultura y Pediatría dependientes de muy diversas instituciones y cuya caótica organización había intentado resolver la Ley de Sanidad Infantil y Maternal promulgada unos meses antes. ${ }^{28} \mathrm{Y}$ las personas encargadas de

\footnotetext{
${ }^{24}$ Según estas mismas estadísticas, el máximo de matronas formadas en un mismo curso académico (1947-48) fue de 26, pero se trató de una clara excepción, pocos años se rebasó la decena de alumnas: A. Arbelo Curbelo, "Medical audit de la Escuela Nacional de Puericultura», en Escuela Nacional de Puericultura 1926-1976. Cincuenta aniversario de la actividad docente y asistencial (Madrid: Ministerio de Sanidad y Seguridad Social, Subsecretaría de la Salud, 1978), 185.

25 Salazar Agulló et al., «Madres y enfermeras», 2.

${ }^{26}$ Ministerio de la Gobernación, «Orden de 20 de diciembre de 1941 por la que se crea la enseñanza de Puericultura para las madres lactantes», Boletín Oficial del Estado, n. 355 (1941): 9993.

${ }_{27}$ Enrique Álvarez Romero, Higiene Infantil e Instructoras de Sanidad (Valladolid: Imprenta Castellana-Ministerio del Interior, Jefatura del Servicio Nacional de Sanidad- Sección de Maternología, Puericultura e Higiene Escolar, Publicaciones «Al servicio de España y del niño español n. ${ }^{\circ}$ 13, (1939), 5. 28 «Ley de 12 de julio de 1941 de Sanidad Infantil y Maternal», Boletín Oficial del Estado, 28 de julio de 1941.
} 
instruir a las madres (en ningún momento aparece el padre como posible partícipe de esta actividad) eran, cómo no, los médicos, pero también las instructoras de Sanidad o las enfermeras visitadoras, entre las que había un buen número de matronas. ${ }^{29}$

A las madres que acudían a los cursos se les entregaba un folleto editado por la sección de Puericultura, Maternología e Higiene Escolar de la Dirección General de Sanidad. El curso constaba de cinco clases teóricas y dos prácticas. La famosa frase de «El Caudillo quiere 40 millones de españoles» se usó como colofón del folleto, depositando en las madres todo el peso de la responsabilidad de la regeneración de la población española.

\section{LA INTRODUCCIÓN DEL PARTO SIN DOLOR EN ESPAÑA: UNA CUESTIÓN DE GÉNERO Y JERAQUÍA}

¿Sabéis por qué la madre ama tanto a sus hijos? Porque es amor arrancado por el dolor ¡Ay el dolor físico de la madre! Que los médicos no lo supriman jamás. Suprímanse todos menos los del alumbramiento. ${ }^{30}$

Este deseo, enunciado en un librito editado por la Falange en 1940, llegaba tarde. A pesar de que, durante siglos, el dolor en el parto se había visto como algo inevitable, ya a finales del siglo XIX, algunas clínicas españolas ofrecían técnicas anestésicas para suprimir el dolor cuando se daba a luz. ${ }^{31}$ Inicialmente, el uso de la anestesia en el parto solo se ofertaba en las ciudades, en consultorios privados y que, por tanto, era una opción solo para mujeres de clases sociales elevadas. La aplicación de la anestesia implicaba un parto hospitalario, una opción minoritaria a principios de siglo en España, pero que iba a ir ganado terreno, poco a poco, a lo largo de la centuria.

\footnotetext{
29 Sobre las enfermeras visitadoras puede consultarse: Josep Bernabeu Mestre y Encarna Gascón Pérez, «El papel de la enfermería en el desarrollo de la salud pública española (1923-1935): la visitadora sanitaria», Dynamis 15 (1995): 151-176.

30 Falange Española Tradicionalista y de las JONS, ¡Madres! La educación maternal (Madrid: Ediciones Auxilio Social, 1940), 13.

31 «Solteras, casadas, madres. Consultorio Portela», La Justicia, 2 de noviembre de 1895.
} 
También fue en el ámbito de la asistencia privada donde se inició la aplicación de los métodos psicosomáticos de control del dolor en España. ${ }^{32}$ Todo parece indicar que la primera experiencia al respecto tuvo lugar el 14 de septiembre de 1953, en la Clínica Santa Alicia, regentada por el ginecólogo Vital Aza Díaz. Pero no fue Vital Aza quien estuvo involucrado en este parto sino su sobrino político, el también ginecólogo Ángel Hernández Jiménez. ${ }^{33}$ Sin embargo, no sería él quien sacase más provecho de la aplicación del método en nuestro país, sino un médico llamado Álvaro Aguirre de Cárcer.

Los métodos psicofísicos del parto sin dolor se estaban extendiendo con rapidez por Europa, lo que provocó la necesidad de la iglesia a pronunciarse sobre el asunto. Dado que las Sagradas Escrituras habían sentenciado a las mujeres a parir con dolor, había dudas sobre la adecuación de su uso a la moralidad cristiana. El ocho de enero de 1956, Pío XII, ante una audiencia de 700 ginecólogos, dio su beneplácito al uso del método: "Si una nueva técnica le evita los sufrimientos del parto o los atenúa, la madre puede aceptarla sin ningún escrúpulo de conciencia, pero no está obligada a ello». ${ }^{34}$ En la España católica de mediados del siglo xx, el discurso de Pío XII sirvió como detonante para promocionar este tipo de técnicas entre la población femenina y los profesionales sanitarios:

En España, la «moda» de estos procedimientos se inicia el día 8 de mayo de 1956, cuando Su Santidad Pío XII se dirige a los tocólogos del mundo entero, reunidos en parte en Roma. Hoy, a fines de 1962, la moda ha pasado y el método puede valorarse sin pasión. ${ }^{35}$

Efectivamente, Aguirre de Cárcer aseguraba que hasta dicha alocución papal tan solo había preparado cinco o seis mujeres, aunque es un

\footnotetext{
32 Lo mismo había sucedido un año antes, de la mano de Fernand Lamaze, en Francia, país desde el que se extendió la práctica a España: Marilène Vuille, «L'Obstétrique sous influence: émergence de l'accouchement sans douleur en France et en Suisse dans les années 1950", Revue d'histoire moderne \& contemporaine 64, n. ${ }^{\circ}$ (2017): 116-149.

33 Arregui, «Psicoprofilaxis y educación maternal», 63.

34 «Importante discurso de Su Santidad el Papa, respecto al parto sin dolor», Matronas 4, n. 1 (1956): 35.

35 Francisco Orengo Díaz del Castillo, Obstetricia para matronas (Madrid: Isidoro Aguirrebeña editor, 1963), 241. Francisco Orengo fue ginecólogo y director de la Escuela de Matronas de Santa Cristina de Madrid durante muchos años. También era el director de la revista Matronas, editada entre 1953 y 1976 y órgano de expresión de la Escuela de Matronas de Santa Cristina, una publicación de gran repercusión entre las matronas rurales de la época.
} 
dato que no debería haberle sorprendido, porque él mismo se había iniciado en el método tan solo unos meses antes:

En el mes de junio de 1955 fui pensionado por el Gobierno francés para realizar un viaje de estudios por Francia. Mi misión era ampliar mis conocimientos en Medicina Reeducativa. Poco antes de salir, y como tenía ya conocimiento de la existencia del llamado "parto si dolor», pregunté a mi antiguo maestro el profesor García Orcoyen (y lo llamo así, porque no en vano estuve cuatro años a su lado en la Cátedra y Servicio de Ginecología y Obstetricia de la Facultad) si consideraba interesante que estudiase este sistema de preparación al parto. Me dijo que sí, y así lo hice. ${ }^{36}$

El interés inicial que despertaron, en algunos médicos, las técnicas de control del dolor en el parto, se relacionaba, fundamentalmente, con sus posibilidades de aplicación a las embarazadas que acudían a sus consultas privadas, o con el deseo de no perder la autoridad dentro del nuevo campo:

La colaboración entre la matrona y el tocólogo resulta una vez más indispensable en el método psicoprofiláctico. Han de aplaudirse los cursillos como el que el Dr. Abad Colomer está dando a las matronas valencianas. En ellos han de sentarse las bases de una división de trabajo que ha de rendir los mejores frutos. ${ }^{37}$

Sin embargo, hubo otro personaje en la historia «del parto sin dolor» cuyo objetivo era conseguir que el método llegase a todas las mujeres de manera gratuita, fuesen de la clase social que fuesen. Se trataba de una matrona y se llamaba Consuelo Ruiz Vélez-Frías, que antes de la alocución del papa ya instruía a mujeres de manera gratuita en la Casa de Salud de Santa Cristina de Madrid. Para poder formarse en el método, había utilizado sus ahorros y sus vacaciones y había marchado a Francia para aprender. Además, tuvo la osadía de transgredir el orden patriarcal escribiendo el primer libro español sobre el parto sin dolor.

\footnotetext{
36 Álvaro Aguirre de Cárcer, «Dos años de experiencia en la preparación al parto», en Conferencias pronunciadas en la Sociedad Ginecológica de Madrid los días 18 y 25 de junio de 1957, (Madrid: Tipografía Flórez), 3.

${ }^{37}$ Francisco Orengo Díaz del Castillo, «Editoriales. El parto sin dolor», Matronas 4, n. ${ }^{\circ}$ (1956): 51-52.
} 
Efectivamente, en 1955, un par de meses antes de la alocución papal, se publicaron dos libros que llevaban exactamente el mismo título: El parto sin dolor: uno estaba firmado por la matrona Consuelo Ruiz Vélez-Frías y el otro por el médico Álvaro Aguirre de Cárcer.

¿Fue pura coincidencia que ambos se decidieran a escribir un libro sobre el mismo tema, con el mismo título y en el mismo año? Consuelo Ruiz aseguraba que el suyo había sido el primer libro en publicarse y que, precisamente esta osadía, le había salido muy cara a nivel profesional:

En la Sociedad Ginecológica hubo una reunión en la que se discutió qué hacer con una matrona que había escrito un libro, qué castigo imponer a tamaña osadía. En tiempos de la Inquisición me hubiera costado ir a la hoguera, pero aunque los tiempos fueran otros, un castigo se me debía imponer, no era suficiente burlarse sentenciando: ii¿Qué una matrona ha publicado un libro?!! ¡Valiente libro será! ¡Como si un asno se pusiera a tocar el piano! La ciencia tenía que escarmentar a la autora de tal desacato.

No me azotaron, ni me cortaron el pelo, ni siquiera me hicieron tragar aceite de ricino, ni barrer las calles.

Silenciosa y educadamente, me expulsaron de mi trabajo en el SOE y en todas las sociedades del Seguro Libre a las que pertenecía $[\ldots] . .38$

Para comprobar si lo que decía la matrona era cierto, se ha procedido a consultar los expedientes de censura editorial de ambos libros que se conservan en el AGA. Pasar por el trámite de la censura era, en esos momentos, un trámite de obligado cumplimiento para cualquier obra que se quisiese publicar. En 1955, la antigua «Sección de Censura de Publicaciones» de la Delegación Nacional de Propaganda del Ministerio de la Gobernación había pasado a denominarse, eufemísticamente, «Inspección de Libros» y los censores eran llamados «lectores», que trabajaban a las órdenes del nuevo Ministerio de Información y Turismo. ${ }^{39}$

\footnotetext{
38 Consuelo Ruiz Vélez-Frías, «La sabiduría silenciada», en Hilando fino. Mujeres, un viaje en común, (Barcelona: Icaria, 2007), 233-277.

39 El Ministerio de Información y Turismo se había creado por el Decreto-Ley de 19 de julio de 1951, por la segregación de algunos departamentos que antes dependían del Ministerio de la Gobernación: «Decreto-Ley de 19 de julio de 1951 por el que se reorganiza la Administración Central del Estado»,
} 
En el expediente de censura del libro de Consuelo Ruiz figura que la solicitud fue presentada por Juan Bachs, dueño de la editorial Enciclopédica, el día 10 de noviembre de 1955. La tirada inicial, de 3.000 ejemplares, tenía un coste de 30 pesetas. ${ }^{40}$

Por su parte, Aguirre de Cárcer presentó por sí mismo la solicitud dos días más tarde y en el expediente se señalaba que se trataba de una autoedición de 2.000 ejemplares. En dicho expediente hay una anotación, a lápiz y manuscrita, en la esquina superior derecha: «Muy urgente», probablemente realizada por el funcionario a instancias del peticionario. ${ }^{41}$

¿Por qué tenía tanta prisa Aguirre de Cárcer? ¿Acaso temía que la matrona pudiese hacerle la competencia? Esta misma reflexión se hacía Consuelo Ruiz muchos años más tarde: «¡Parece mentira que una persona tan insignificante, tan humilde, una simple "matrona sin derechos", pudiera hacerles sombra»!42

Es evidente que el libro de Consuelo Ruiz llevaba más tiempo escrito que el de Aguirre de Cárcer. Ella había presentado su obra, ya editada, en el Primer Congreso Nacional de Matronas que acababa de celebrarse en Madrid, del 26 al 29 de octubre de $1955^{43}$ y había conseguido una editorial que quisiera publicarlo, un proceso que, evidentemente, era mucho más largo que la autoedición. ${ }^{44}$ Resulta un tanto sorprendente el afán del ginecólogo por no quedarse rezagado en la carrera por la edición.

Boletín Oficial del Estado, n. 201 (1951): 3446. Para saber más sobre la historia de los expedientes de censura puede consultarse: Daniel Gozalbo Gimeno, «Historia archivística de los expedientes de censura editorial (1942-2017)», Creneida 5 (2017): 8-34.

40 «Expediente n. ${ }^{\circ}$ 5.812-55. Solicitud de autorización para imprimir la obra El parto sin dolor», de Consuelo Ruiz Vélez-Frías, 10 de noviembre de 1955, Cultura, expedientes de censura editorial, caja 21/11271, AGA.

41 «Expediente n. ${ }^{0}$ 5.845-55. Solicitud de autorización para imprimir la obra El parto sin dolor», de Álvaro Aguirre de Cárcer, 12 de noviembre de 1955, Cultura, expedientes de censura editorial, caja 21/11272, AGA.

42 Consuelo Ruiz Vélez-Frías, Parir sin miedo. El legado de Consuelo Ruiz (Tenerife: Editorial OBSTARE, 2010): 51.

43 «Retribución decorosa piden las matronas españolas. Un método de "parto si dolor" se expone en el congreso», Ya, 28 de octubre de 1955.

${ }^{44} \mathrm{El}$ original que se conserva junto al expediente de censura, de la obra de Aguirre de Cárcer, no tiene la apariencia externa de la que tiene la primera edición, sino la de unas galeradas. La cubierta es de tapa blanda y pegada mientras que en la edición final la cubierta es de tapa dura con las hojas cosidas. 
Especialmente si tenemos en cuenta las pocas posibilidades de competir con él que tenía la matrona, dada su triple condición de mujer, con una profesión considerada subalterna y con escasos recursos económicos, en una sociedad tan androcéntrica y clasista como la que caracterizaba a la España de mediados del siglo xx.

Además, el público al que iban dirigidos ambos libros no era el mismo. Consuelo Ruiz había escrito un libro destinado fundamentalmente a las embarazadas, mientras que el Álvaro Aguirre iba dirigido, teóricamente, a profesionales sanitarios:

Yo me tracé una línea de conducta y he procurado seguirla hasta este momento. Yo me iba a entender con los médicos y en especial con los tocólogos; no se me ocurría entablar un diálogo directo con el público. Mi libro iba dirigido al médico ¿Qué resultaba muy sencillo? ¿Qué las mujeres lo podían leer? De acuerdo, pero yo me dirigía al médico. ${ }^{45}$

La noticia de que la matrona estaba a punto de publicar su libro sobre el parto sin dolor le pudo llegar a Aguirre de Cárcer por diversas vías. A través de Jesús García Orcoyen, por ejemplo, que manejaba en la sombra los hilos del Colegio de Matronas y había intervenido activamente en la organización del I Congreso Nacional de Matronas. De hecho, fue él quien animó a Aguirre de Cárcer a emprender su viaje de estudios como hemos visto con anterioridad.

También pudo informarle su colega Puebla Ridaura, jefe de Consuelo en el Equipo Tocológico de Urgencia n. ${ }^{\circ} 1$ de Madrid y consocio de Aguirre de Cárcer en la Sociedad Ginecológica Española. Consuelo Ruiz había pedido a Puebla Ridaura que le escribiese el prólogo de su obra, tarea a la que accedió el ginecólogo a pesar de que en él afirmaba: «Siento que se haya fijado en mí - atención que atribuyo a su deseo de dar al jefe una supremacía que, más que un halago me supone una tortura- y no haya recurrido a firmas más autorizadas y competentes con las que también tiene relación directa».46

\footnotetext{
45 Álvaro Aguirre de Cárcer, «Dos años de experiencia en la preparación al parto», en Conferencias pronunciadas en la Sociedad Ginecológica de Madrid los días 18 y 25 de junio de 1957, (Madrid: Tipografía Flórez), 3. Con esta afirmación, parece como si Álvaro Aguirre de Cárcer se estuviese disculpando por haber escrito un libro poco académico.

${ }^{46}$ Consuelo Ruiz Vélez-Frías, El Parto sin dolor, (Madrid: Editorial Enciclopédica, 1955), 5.
} 
Según Amaya García Arregui, Consuelo era «muy popular entre las matronas pero ignorada por la clase médica». ${ }^{47}$ Esta afirmación no concuerda con otras fuentes, incluida la nota anterior de Puebla Ridaura en la introducción del libro de Consuelo Ruiz. En todo caso, probablemente no se trataba de una ignorancia real, sino fingida, premeditada y estratégica por parte de algunos profesionales médicos. Y no de todos: en la revista Matronas, que editaba el doctor Francisco Orengo se publicaron, en esos años, numerosos artículos escritos por la matrona y noticias en las que ella era la protagonista, como veremos más adelante.

De cualquier manera, ambas publicaciones tuvieron mucho éxito. Tan solo unos meses más tarde, tanto Consuelo Ruiz como Aguirre de Cárcer, solicitaban a la «Inspección de Libros», del Ministerio de Información y Turismo, la autorización para publicar una segunda edición de sus respectivas obras, por haberse agotado la edición anterior. Mientras Álvaro Aguirre lo hizo el 13 de marzo de 1956, Consuelo lo solicitaba el 15 de junio de ese mismo año. Esto supone que, en tan solo siete meses, se habían vendido más de 5.000 libros sobre el "parto sin dolor» en España, lo que corrobora la moda a la que hacía referencia Francisco Orengo.

A pesar de haber bebido de las mismas fuentes, los métodos de ambos eran muy diferentes. Mientras Aguirre de Cárcer opinaba que el mejor lugar para el desarrollo de los cursos era un gimnasio, Consuelo Ruiz rechazaba la parte de preparación física del método, pensamiento que mantuvo hasta el final de sus días:

Hasta la previa «educación maternal», tenía reminiscencias de instrucción militar, pues la gimnasia a la que se sometía a las embarazadas se semejaba al adiestramiento de los quintos, y su objetivo era el mismo: lograr el grado de disciplina necesario para que los individuos se sometieran, sin hacer preguntas, sin rechistar, a cuanto se les ordenara. ${ }^{48}$

Un gran número de matronas se interesaron por adquirir conocimientos sobre el método psicoprofiláctico a partir del momento en que el papa Pío XII dio el visto bueno a su utilización en los partos. Este interés

\footnotetext{
47 Arregui, «Psicoprofilaxis y educación maternal», 100.

48 Ruiz Vélez-Frías, Parir sin miedo, 37.
} 
fue, al menos en parte, fruto de la propaganda que se hizo de ellos en la revista Matronas. ${ }^{49}$ Casi en cada número de los publicados entre los años 1956 y 1970 incluyó algún artículo o alguna reseña de un libro que trataba el tema. Muchas matronas realizaron consultas a Francisco Orengo, sobre el tema, en la sección de «Cartas al director» de la revista. El mismo interés encontramos tras el inicio de la publicación, en 1959, de la revista Súrgere. Sin embargo, Consuelo Ruiz pagó cara su osadía de intentar ponerse al mismo nivel que un médico, pero para comprenderlo, debemos saber algo más sobre ella, antes de ser matrona: «Hubo una época, exactamente cuando yo tenía veinticinco años, en la que a muchísima gente nos pareció que el cielo se hundía sobre nuestras cabezas».50 Así relataba Consuelo Ruiz Vélez-Frías el sentimiento que le produjo la instauración de la Dictadura después de que acabase la Guerra Civil. «Yo era, entonces, una jovencita enclenque, pequeñaja e insignificante y me costó mucho salir adelante».51

No es de extrañar el pavor que experimentó esta mujer que había sido miembro del Socorro Rojo Internacional, y que se había significado durante la guerra impartiendo mítines feministas y escribiendo numerosos artículos en la revista Ayuda que firmaba con el pseudónimo de Elisa Risco. Formaba parte de un grupo al que también pertenecía Matilde Landa. ${ }^{52}$ Afortunadamente la utilización del pseudónimo Elisa Risco la ayudó a salvarse del cruel destino compartido por muchos de los que habían luchado defendiendo la República. ${ }^{53}$

\footnotetext{
${ }_{49}$ La revista Matronas fue el órgano de expresión de la Casa de Salud de Santa Cristina de Madrid, dirigida y editada por Francisco Orengo, director de la escuela de matronas del centro.

50 Ruiz Vélez-Frías, «La sabiduría silenciada», 236.

${ }^{51}$ Ruiz Vélez-Frías, «La sabiduría silenciada», 236. Este dato queda confirmado por el reconocimiento médico que le hicieron con motivo de su desempeño laboral dentro de la sanidad pública. En esos momentos, Consuelo, que ya tenía 51 años, medía 148 centímetros, pesaba 41 kilogramos y el médico la clasificaba como leptosomática. A pesar de que presentaba una «hipertensión por arterioesclerosis bien controlada», fue considerada apta para el servicio: Consuelo Ruiz Vélez-Frías, «Hoja clínica de su expediente personal», 1 de junio de 1966, expediente personal de Consuelo Ruiz-Vélez Frías, 12, Expedientes personales por orden alfabético, Archivo del Instituto de Gestión Sanitaria (Archivo INGESA).
}

52 Matilde Landa Vaz fue una destacada figura de la resistencia antifranquista, miembro del PCE y del Socorro Rojo Internacional, se suicidó en la prisión de mujeres de Palma de Mallorca tras ser apresada al final de la Guerra Civil. Para saber más sobre ella se puede consultar: David Ginard Féron. Matilde Landa. De la Institución Libre de Enseñanza a las prisiones franquistas (Barcelona: Flor del Viento Ediciones, 2005).

${ }_{53}$ En el Centro Documental de la Memoria Histórica (CDMH) existe un expediente incoado contra ella por el Tribunal Especial para la Represión de la Masonería y el Comunismo. El expediente no 
Como tantas personas de la época, nuestra protagonista tuvo que aguzar el ingenio para poder prosperar y dejar atrás las penurias de la postguerra. Tras realizar sus estudios de matrona consiguió un puesto en la Beneficencia Municipal de Madrid y, el 9 de junio de 1953, fue nombrada matrona del Seguro Obligatorio de Enfermedad (SOE) de la capital, a pesar de que ya llevaba prestando estos servicios desde el primero de junio de 1951. ${ }^{54}$ También comenzó a trabajar para varias compañías médico-farmacéuticas que prestaban sus servicios al denominado «seguro libre».55

Tras la presentación de su libro en el congreso de matronas de 1955, todo parecía ir sobre ruedas, por una vez, en la vida de Consuelo Ruiz. El 14 de enero de 1956 recibió un homenaje «justo tributo a su meritísima labor, a su gran celo para documentarse (sin temer para ello desplazarse al extranjero) del máximo acontecimiento de actualidad en lo que a nuestra profesión afecta: "El parto sin dolor"». Al acto, organizado por varias de sus compañeras de profesión, también asistieron algunos tocólogos. ${ }^{56}$

El 31 de diciembre de 1956 causó baja como matrona del SOE, sin que aparezcan en su expediente los motivos de su baja que, como ya se ha comentado antes, ella aseguraba que fue un despido fruto de la venganza de algunos ginecólogos. ${ }^{57}$

empezó a instruirse hasta una fecha tan tardía como el 7 de noviembre de 1960 y en él se encuentran datos sobre sus actividades propagandísticas en diversos mítines y publicaciones periódicas de la época. Debía ser muy buena oradora «a mí me querían sacar en hombros, sin duda por ser la más pequeña» dice en una de las cartas transcritas en su expediente. Este fue archivado el 23 de septiembre de 1963 por «ignorado paradero», ya que el único dato con que contaban las fuerzas de orden público era que estaba domiciliada en Madrid: Elisa Risco, «Expediente de Elisa Risco en el Tribunal Especial para la Represión de la Masonería y el Comunismo, juzgado especial n. ${ }^{\circ}$ 3», 10 de mayo de 1937 a 23 de septiembre de 1960, signatura CDMH_TERMC_C-EXP60774, CDMH.

${ }^{54}$ Consuelo Ruiz Vélez-Frías, «Nombramiento como matrona interina del Seguro obligatorio de Enfermedad», 9 de junio de 1953, expediente personal de Consuelo Ruiz-Vélez Frías, 2, Serie de expedientes personales por orden alfabético, Archivo INGESA.

${ }_{55}$ Para saber más sobre el desarrollo de los seguros de salud en España puede consultarse: Jerònia Pons Pons y Margarita Vilar Rodríguez, El seguro de salud privado y público en España. Su análisis en perspectiva histórica (Zaragoza: Prensas de la Universidad de Zaragoza, 2014).

${ }_{56}$ M. ${ }^{a}$ Rosa Virseda, «Homenaje a Consuelo Ruiz Vélez-Frías», Matronas 4, n. 2 (1956): 78-79.

57 Consuelo Ruiz Vélez-Frías, «Baja como matrona interina del Seguro obligatorio de Enfermedad», 19 de enero de 1957, expediente personal de Consuelo Ruiz-Vélez Frías, 6, Serie de expedientes personales por orden alfabético, Archivo INGESA. 
Este pensamiento de la matrona no era descabellado. En esos años las matronas que trabajaban en los equipos tocológicos dependían absolutamente del tocólogo titular, de tal manera que eran estos los que las nombraban y despedían sin necesidad de dar explicaciones. Cuando un tocólogo se jubilaba, por ejemplo, lo más habitual es que el nuevo trajese su propio equipo, quedando las antiguas totalmente desamparadas. ${ }^{58}$

Consuelo Ruiz justificaba su decisión de migrar a su despido, pero esto no concuerda con la documentación pues el cese se produjo en 1956 y su salida de España hacia Sudamérica no tuvo lugar hasta 1960, justo cuando se empieza a instruir el sumario contra Elisa Risco, su alter ego, por el Tribunal para la Represión de la Masonería y el Comunismo. ¿Acaso quisieron asustarla por su pasado comunista? Aunque no lo explica claramente, algo así pudo suceder:

Yo me vi obligada a salir de España con el sambenito de filocomunista, que me pesaba como una losa de plomo. No me atrevía a solicitar ningún otro trabajo, ni tampoco quería verme reducida a fregar escaleras, como alguna colega me auguraba, en justo castigo a mi soberbia de querer mejorar el parto, sin tener la debida categoría para ello. ${ }^{59}$

A su vuelta a España, se registró en el Colegio Oficial de Enfermeras ATS de Madrid el 11 de abril de 1966.60 En junio de ese mismo año estaba prestando servicios como enfermera eventual en el ambulatorio «Pedro González Bueno», en el distrito de San Blas (Madrid), aunque no duró mucho en el puesto, ya que causó baja en 31 de agosto. ${ }^{61}$ Recuperó su plaza de matrona de la Beneficencia Municipal de Madrid y allí, en el equipo

\footnotetext{
58 Para más información sobre la situación de las matronas durante la dictadura franquista puede consultarse: Dolores Ruiz-Berdún «Las dificultades de las matronas en la España rural del siglo xx» en Genealogías de la Reforma Sanitaria en España, ed. José Martínez Pérez y Enrique Perdiguero Gil (Madrid: Los Libros de la Catarata, 2020), 21-62.

59 Ruiz Vélez-Frías, «La sabiduría silenciada», 254.

${ }^{60}$ Consuelo Ruiz Vélez-Frías, «Certificado de colegiación en el Colegio oficial de Enfermeras ATS de Madrid», 16 de mayo de 1966, expediente personal de Consuelo Ruiz-Vélez Frías, p. 10, Serie de expedientes personales por orden alfabético, Archivo INGESA.

61 Servicio de Ordenación Sanitaria, «Bajas Enfermeras Instituciones», 20 de septiembre de 1966, expediente personal de Consuelo Ruiz-Vélez Frías, 10, Serie de expedientes personales por orden alfabético, Archivo INGESA.
} 
Tocoginecológico Municipal de urgencia n. ${ }^{\circ} 1$, estuvo preparando parejas hasta su jubilación forzosa en 1984:

Yo explicaba en el encerado, con tizas de colores, el embarazo, el parto y la lactancia, como si fuesen teoremas, pero no hubo ni una sola matrona que se interesara por seguir mi sistema ni por enterarse de por qué dolía el parto. Puede ser que la preparación les pareciese complicada y poco indicada para la cultura femenina. Era más sencillo ponerles a respirar y a hacer gimnasia, sin explicarles para qué ni por qué. Verdaderamente, yo tampoco sé qué relación tiene la gimnasia con la musculatura uterina, que es de fibra lisa, y no es de extrañar que mis colegas tampoco lo supieran. Un médico había dicho que las embarazadas tenían que hacer gimnasia, el médico es un ser superior que sabe más y eso bastaba. ${ }^{62}$

\section{EL INTERÉS DE LAS MATRONAS EN LA EDUCACIÓN PARA EMBARAZADAS}

Consuelo Ruiz fue un personaje clave para popularizar el parto sin dolor entre el resto de las matronas españolas, pero su partida al extranjero hizo que perdiese el protagonismo que debía haber tenido para la profesión. No hay constancia de ningún otro libro anterior escrito por una matrona a excepción del antecedente de Francisca Iracheta en el siglo XIX. ${ }^{63}$

De hecho, los libros destinados a la formación de las matronas españolas siempre los habían escrito los médicos. El mismo monopolio médico se produjo en el diseño de los planes de estudio de las matronas y en la dirección de sus enseñanzas hasta bien entrados los años noventa. La última regulación de la carrera de matrona, antes de su desaparición como estudios independientes, se había producido en $1904 .{ }^{64}$ El escueto

\footnotetext{
62 Ruiz Vélez-Frías, «La sabiduría silenciada», 255-256.

63 Teresa Ortiz Gómez, «De matrona a matrona. Francisca Iracheta y la divulgación de la ciencia obstétrica en España en 1870», Arenal 6, n. 1 (1999): 183-195.

64 «Real decreto, de 10 de agosto de 1904, reorganizando los estudios de la carrera de Practicantes y la de Matronas», Gaceta de Madrid, 12 de agosto de 1904. Sobre este tema puede consultarse: Dolores Ruiz-Berdún, «Pilar Primo de Rivera y la reorganización de las carreras auxiliares sanitarias tras la Guerra Civil», en La tutela imperfecta. Biología y Farmacia en la España del primer franquismo, eds.
} 
programa del Real Decreto era puramente biologicista y no contemplaba ningún contenido educativo hacia las embarazadas. En 1957 la carrera de matrona pasó a ser una especialidad de los estudios de Ayudante Técnico Sanitario (ATS), una nueva figura creada unos años antes y que agrupaba las carreras de practicante y de enfermera. ${ }^{65}$ Aunque la especialidad iba a ser inicialmente de dos años y podría ser cursada en la escuelas femeninas y masculinas, finalmente se limitó a un curso de duración y destinado únicamente a las ATS femeninas. La única referencia a la educación de las embarazadas en el programa aparecía en el tema 38, que estaba dedicado a la «Preparación psicológica de la embarazada. Diversos métodos de analgesia y anestesia en el parto».66

Con respecto a los libros de texto, las primeras referencias a la preparación específica para el parto las encontramos en la tercera edición del libro Obstetricia para matronas de Francisco Orengo. ${ }^{67}$ En el capítulo doce, titulado "La preparación psicofísica de la embarazada», el autor establecía la importancia de este método para las matronas: «La matrona solo dispone de la preparación psicofísica y debe aprovecharla. El médico puede añadir inmensos recursos farmacológicos y técnicos». ${ }^{68}$

Esta frase tan sencilla, tiene, no obstante, diversas lecturas. Por un lado, precisamente la imposición de que las matronas no pudieran utilizar

Antonio González Bueno y Alfredo Baratas Díaz (Madrid: Consejo Superior de Investigaciones Científicas, 2013), 81-100.

${ }_{65}$ «Orden, de 4 de julio de 1955, por la que se dictan normas para la nueva organización de los estudios de Ayudantes Técnicos Sanitarios», Boletín Oficial del Estado, 2 de agosto de 1955. En dicha orden aparece el plan de estudios, cuyo análisis es muy interesante para comprender la diferente visión que tenía la dictadura franquista con respecto a la educación de hombres y mujeres. Las futuras ATS femeninas debían realizar sus estudios en la modalidad de internado, cosa que no sucedía con sus compañeros varones. Otra diferencia significativa era que mientras en las escuelas de ATS femeninas se incluía durante los tres cursos una hora semanal de la disciplina «Enseñanza de Hogar», en la de ATS masculinos esa hora se destinaba al aprendizaje de las nociones de autopsia médico-legal. En ambos casos se incluían 30 horas tanto de religión como de moral profesional y una hora semanal de formación política.

66 «Orden, de 23 de febrero de 1957, por la que se aprueba el programa para las enseñanzas de especialización de asistencia obstétrica (Matrona) para los Ayudantes Técnicos Sanitarios Femeninos», Boletín Oficial del Estado, 5 de abril de 1957.

${ }^{67}$ Obstetricia para matronas probablemente haya sido el libro más utilizado durante el siglo xx por las matronas españolas, ya que fue el manual que se utilizó durante más tiempo en la Escuela de Matronas de la Casa de Salud de Santa Cristina de Madrid. Su primera edición data de 1949.

${ }^{68}$ Francisco Orengo Díaz del Castillo. Obstetricia para matronas (Madrid: Isidoro Aguirrebeña editor, 1963), 241. 
más que recursos «naturales» (cambios de posición, termoterapia, masajes y ungüentos) fue la manera de ir desplazando a las mujeres del monopolio que habían mantenido durante siglos sobre los partos. ${ }^{69} \mathrm{Al}$ mismo tiempo, los cirujanos comenzaron popularizando el fórceps y fueron añadiendo a su arsenal todo tipo de instrumentos, técnicas y medicamentos, apoderándose, incluso, de algunos recursos que habían sido tradicionalmente utilizados por las parteras como el cornezuelo de centeno. ${ }^{70}$ La frase también servía de recordatorio a las matronas de su papel subordinado al del médico, un recurso histórico presente, prácticamente, en todos los libros de texto escritos por ginecólogos para la formación de las matronas..$^{71}$ Por último, también era un intento de convencer a las matronas para que utilizasen el método, lo cual podía suponer un beneficio tanto para la gestante como para todos los profesionales que la atendiesen en su parto.

El capítulo era muy escueto y claramente insuficiente para poder aplicarlo a la práctica, pero para eso ya estaban los cursillos de perfeccionamiento que empezaron a organizarse en Santa Cristina y cuyo director era, también, Francisco Orengo. Salvo algunas excepciones, la mayoría de las cursillistas que acudían a formarse provenían del ámbito rural, donde las posibilidades de actualización profesional eran muy limitadas. Dentro del programa de estos cursos de formación continuada, se incluía invariablemente una clase práctica de Psicoprofilaxis y diversas conferencias sobre el mismo tema. ${ }^{72}$

Una de las alumnas formada en otra de las escuelas de matronas de Madrid, la de la Cruz Roja, refiere no haber recibido formación específica en psicoprofilaxis. Quienes querían aprender debían hacerlo por su cuenta:

\footnotetext{
${ }^{69}$ Hay abundante bibliografía sobre este proceso de expropiación que no fue exclusivo de España: Véase, por ejemplo para Francia: Nathalie Sage Pranchère, "La résistance d'une profession», en L'école des sages femmes: Naissance d'un corps professionel, 1786-1917 (Tours: OpenEdition Books, 2017). https://doi.org/10.4000/books.pufr.13223. Y para Inglaterra: Jean Donnison, Midwives and medical men. A History of Interprofessional Rivalries and Women's Rights (London: Heinemann, 1977), 31.

70 Dolores Ruiz-Berdún, «Evolución de la fabricación, comercialización y uso de los derivados del cornezuelo de centeno en Obstetricia», en De la botica de El Escorial a la industria farmacéutica. En torno al medicamento, ed. Alberto Gomis Blanco y Raúl Rodríguez Nozal (Alcalá de Henares: Servicio de Publicaciones de la Universidad de Alcalá, 2015): 334-335.

71 Ángela Torres Díaz, «Un manual para el adoctrinamiento de las matronas», Temperamentvm 13 (2017). https://ciberindex.com/index.php/t/article/view/e11600 (consultado el 20-3-2021).

72 «IV Curso de perfeccionamiento para Matronas», Matronas 23, n. ${ }^{\circ}$ (1975): 25-27.
} 
Preparación al parto no había. Yo me fui a formar, a la vez que hacía matrona, me iba por las tardes a la consulta... a una clínica que tenía el doctor Sanchís que hacía preparación al parto y era de la escuela de la psicoprofilaxis obstétrica. Le pedimos por favor que nos admitiera y luego ya al finalizar fui con este, ¿cómo se llama? el de La Paz, ay, no me acuerdo. Hasta que ya hice yo sofrología por mi cuenta, pero ya de matrona. ${ }^{73}$

Nuestra informante, Hortensia García Pacheco (H.G.P.), realizó primero varios cursos con Aguirre de Cárcer y posteriormente los tres niveles de Preparación Sofrológica a la Maternidad (básico, medio y superior), de la Sección Española de la Federación Mundial de Sofrología. Para ello tuvo que desplazarse a Barcelona para asistir a los cursos de la Escuela Española de Sofrología, ${ }^{74}$ cuyo presidente en esos momentos era Mariano Espinosa. Una de las directoras del curso era la matrona y fisioterapeuta Gloria Sebastiá Gracia, ${ }^{75}$ quien había incorporado la formación en educación maternal para las matronas que hacían la especialidad en el Hospital Clínico de Barcelona en los años ochenta. ${ }^{76}$

Otra muestra del creciente interés de las matronas por participar en la educación de las madres se constata en el protagonismo que fue adquiriendo dentro de las reuniones científicas en las que participaban u organizaban ellas mismas. Ya se ha mencionado que, en el Primer Congreso Nacional de Matronas, de 1955, Consuelo Ruiz Vélez-Frías presentó el método del parto sin dolor. También lo hicieron en la V Reunión de Sanitarios Españoles, ${ }^{77}$ y en todos los congresos nacionales e internacionales celebrados a partir de ese momento. ${ }^{78}$ Las matronas eran conscientes de que el cambio que se estaba produciendo en el escenario de atención

\footnotetext{
${ }^{73}$ Entrevista a H.G.P., matrona jubilada, realizada el 10 de octubre de 2018.

74 Sobre el desarrollo de la Sofrología en España: Ángel González de Pablos, «Las nuevas ciencias del espíritu del siglo xx: La aparición de la sofrología en España a inicios del segundo franquismo», Cultura Psi, n. ${ }^{\circ} 6$ (2016): 91-117.

75 Diplomas de los cursos básico, medio y avanzado de la Preparación Sofrológica a la Maternidad, colección particular H.G.P. Por supuesto, todos los gastos corrían a costa de la interesada.

76 J. M. Miguez, «De la maternidad vigilada a la maternidad vigilante. Cómo son y por qué son necesarios los cursillos de preparación al parto», La Vanguardia, 1 de agosto de 1981: 34.

77 Julieta Samaniego, «Labor del consejo», Súrgere 1, n. 3 (1959): 9.

78 En el discurso inaugural del II Congreso Nacional de Matronas, celebrado en Barcelona del 28 de septiembre al 2 de octubre de 1959, Juan Boch Marín recordaba que la matrona era «la consejera
} 
al parto, desde la casa al hospital, iba a disminuir la necesidad numérica de matronas. No quedaba más remedio que ir incorporando nuevas competencias si no querían desaparecer como profesión.

La educación maternal fue, precisamente, la temática principal del V Congreso Nacional de Matronas, ${ }^{79}$ cuyo lema era «La matrona en la educación maternal».

En esta etapa final del franquismo, las matronas seguían estando fuertemente tuteladas por la profesión médica. En los cuatro días que duró el congreso hubo 6 conferencias, todas impartidas por médicos y numerosas ponencias también a cargo de estos profesionales (tabla 1).

Tabla 1. Conferencias y ponencias relacionadas con la educación maternal impartidas en el V Congreso Nacional de Matronas

\begin{tabular}{|c|c|c|}
\hline $\begin{array}{c}\text { Conferenciante o } \\
\text { ponente }\end{array}$ & Cargo & Título de la conferencia \\
\hline $\begin{array}{l}\text { Vicente } \\
\text { Salvatierra Mateu } \\
\text { (conferencia) }\end{array}$ & $\begin{array}{l}\text { Catedrático de Obstetricia } \\
\text { y Ginecología de la } \\
\text { Facultad de Medicina de } \\
\text { Granada }\end{array}$ & $\begin{array}{l}\text { «Concepto de la educación } \\
\text { maternal: preparación de la mujer } \\
\text { para la maternidad y el parto, y en } \\
\text { general para la vida sexual y } \\
\text { conyugal» }\end{array}$ \\
\hline (ponencia) & $\begin{array}{l}\text { Representante del Colegio } \\
\text { provincial de matronas de } \\
\text { Alicante }\end{array}$ & $\begin{array}{l}\text { «Sopología» (sic, probablemente } \\
\text { sería Sofrología) }\end{array}$ \\
\hline $\begin{array}{l}\text { Rosa Mercadé } \\
\text { (ponencia) }\end{array}$ & $\begin{array}{l}\text { Presidenta del Colegio } \\
\text { Provincial de Matronas de } \\
\text { Barcelona }\end{array}$ & $\begin{array}{l}\text { «Concepto de Educación } \\
\text { maternal» }\end{array}$ \\
\hline $\begin{array}{l}\text { Antonio Martínez } \\
\text { Valverde } \\
\text { (conferencia) }\end{array}$ & Médico de Granada & «Educación maternal pediátrica» \\
\hline $\begin{array}{l}\text { Antonio Galdó } \\
\text { Villegas } \\
\text { (conferencia) }\end{array}$ & $\begin{array}{l}\text { Catedrático de Pediatría } \\
\text { de la Facultad de } \\
\text { Medicina de Granada }\end{array}$ & «Educación maternal neonatal» \\
\hline
\end{tabular}

autorizada de la familia en orden a la maternidad»: Juan Bosch Marín, «Discurso inaugural», Súrgere 1, n. ${ }^{\circ} 8$ (1959): 15.

${ }^{79}$ El congreso se celebró en Granada entre el 20 y el 23 de noviembre de 1974. 


\begin{tabular}{|l|l|l|}
\hline \multicolumn{1}{|c|}{$\begin{array}{c}\text { Conferenciante o } \\
\text { ponente }\end{array}$} & \multicolumn{1}{|c|}{ Cargo } & \multicolumn{1}{c|}{ Título de la conferencia } \\
\hline $\begin{array}{l}\text { Álvaro Aguirre de } \\
\text { Cárcer }\end{array}$ & Médico de Madrid & «Educación maternal» \\
\hline $\begin{array}{l}\text { Ángel Escudero } \\
\text { Juan }\end{array}$ & Médico de Valencia & $\begin{array}{l}\text { «Anestesia psicológica } \\
\text { maternofetal» }\end{array}$ \\
\hline $\begin{array}{l}\text { José Martínez } \\
\text { Estrada }\end{array}$ & $\begin{array}{l}\text { Delegado General del } \\
\text { Instituto Nacional de } \\
\text { Previsión }\end{array}$ & $\begin{array}{l}\text { «Educación maternal de la mujer } \\
\text { que trabaja: maternidad, profesión } \\
\text { y legislación» }\end{array}$ \\
\hline
\end{tabular}

Aunque es de suponer que algunas matronas presentasen comunicaciones libres relacionadas con la educación maternal, ninguno de sus nombres figuraba en el programa oficial. ${ }^{80}$ Gracias a que su ponencia fue posteriormente publicada en la revista Súrgere, sabemos que intervino Rosa Mercadé, presidenta del Colegio de Matronas de Barcelona. En su ponencia abogaba por que las matronas no solo se dedicasen a la preparación de las mujeres para el parto y la maternidad, sino para su vida sexual y conyugal, ampliando así el campo de actuación de la profesión. ${ }^{81}$

Consuelo Ruiz, que a esas alturas contaba ya con sesenta años, no asistió, "con gran disgusto», a este congreso tan íntimamente relacionado con su devenir vital y profesional. Probablemente la presencia entre los conferenciantes de la persona a la que consideraba responsable de su postergamiento profesional y su persecución política, Aguirre de Cárcer, influyó en su decisión de no asistir. Habiendo sido pionera en la difusión del método psicoprofiláctico en España y autora del primer libro sobre el tema en nuestro país, lo lógico es que ella hubiese figurado entre los conferenciantes del programa oficial. Pero como suele decirse «nadie es profeta en su tierra», muchas matronas, que habían sucumbido al proceso de subordinación, asumían que sus fuentes de conocimiento debían de provenir de los médicos. Además, la orientación política de Consuelo Ruiz chocaba de frente con la de la junta directiva del consejo de matronas, que era quien dirigía

\footnotetext{
${ }^{80}$ Tan solo aparece el nombre de Flavia Rodríguez en una comunicación titulada «Significación, situaciones y proyección de la Matrona en la Seguridad Social», programa oficial del V Congreso Nacional de Matronas celebrado en Granada en 1974. Fuente: colección particular.

${ }^{81}$ Rosa Mercadé, «Educación maternal», Súrgere 16, n. 193 (1975): 3-6.
} 
la marcha de los congresos organizados por los colegios provinciales. ${ }^{82}$ No obstante, Consuelo envió una comunicación escrita, que sería publicada en la revista Súrgere del mes de enero de 1975, y en la que seguía insistiendo en la importancia de la matrona en los cuidados psicológicos de las madres y en utilizar una metodología alejada de exposiciones excesivamente científicas. ${ }^{83}$ Una de las conclusiones del congreso consistió en solicitar la creación de centros específicos de educación maternal, ya que las instalaciones en las que se desarrollaban los cursillos eran claramente insuficientes. ${ }^{84}$

En los años setenta del siglo xx, la educación a las madres, dentro del sistema sanitario público, tenía su epicentro en las grandes residencias sanitarias franquistas. De esta forma, parte de la responsabilidad de la educación de las embarazadas recayó en las matronas destinadas en las consultas externas de tales centros. El programa que ofertaba la «Ciudad Sanitaria Juan Canalejo» de La Coruña, a finales de los años setenta, era bastante ambicioso. Abarcaba desde la primera consulta de embarazo, donde se informaba a la gestante de la existencia de los cursos y de las ventajas de asistir a ellos, hasta el puerperio tardío. Además de los contenidos habituales de los cursillos (teoría, respiración y técnicas de relajación) se hacían visitas guiadas por las diferentes estancias del servicio materno-infantil (urgencias, habitaciones, paritorios), intentando eliminar el miedo por medio de la desensibilización sistemática.

Estas mismas matronas acudían a la sala de dilatación para recordar los conceptos de la preparación a las mujeres previamente preparadas, o a realizar un cursillo exprés, a las que no lo estaban, con los fundamentos básicos de la relajación y la respiración. Hay que tener en cuenta que en esa época las parturientas no podían contar con acompañante y debían pasar todo el proceso de dilatación y expulsivo y alumbramiento

\footnotetext{
82 Desde el principio de la dictadura, la presidencia de los colegios profesionales de matronas y practicantes habían recaído en miembros de la Falange, una forma más de control que utilizó el dictador Francisco Franco. Hasta 1953, fecha en que se produce la unificación de las titulaciones de enfermeras y practicantes mientras que la de matrona pasó a ser una especialidad, las enfermeras no tuvieron posibilidad de tener colegio profesional. A partir de esa fecha sería Mercedes Milá, que había ejercido las labores de jefa de enfermeras de la División Azul, la presidenta de su colegio: Ruiz-Berdún, «Pilar Primo de Rivera», 98.

${ }_{83}$ Consuelo Ruiz, «Comunicación al V Congreso Nacional de Matronas», Súrgere 16, n. 191 (1975): 3-4.

${ }^{84}$ «Conclusiones del Congreso Nacional de Matronas», Matronas 23, n. ${ }^{\circ}$ (1975): 27.
} 
en soledad, ${ }^{85}$ por lo que el apoyo que este personal les proporcionaba era muy bien recibido. Durante el puerperio hospitalario, además de la atención personalizada, habitación por habitación, para enseñar ejercicios respiratorios y circulatorios, se organizaban dos charlas grupales semanales donde se abordaban aspectos como la higiene del puerperio, las técnicas de lactancia, cuidados del bebé, y los métodos anticonceptivos. ${ }^{86}$ Al mes del parto, las nuevas madres que lo deseasen podían acudir a realizar ejercicios de gimnasia postnatal. Según las propias matronas, su participación en este tipo de programas completaba su «labor asistencial que por sí sola sería menos estimulante, lo que contribuiría al deterioro de la misma». 87

Pero no todas las mujeres tenían facilidad para acudir a los cursos, por lo que, intentando llegar al mayor número de embarazadas posible, en 1976 se introdujo «el método continuo en la educación maternal». Este método era una simplificación de la preparación, con una sola clase tipo, que se repetía cada semana, y en la que «el tocólogo o en su defecto la matrona», hacía una breve exposición de nociones anatómicas y fisiológicas, el desarrollo feto-placentario, las etapas del parto y «se insiste en considerar a la contracción como un trabajo muscular, exponiendo el origen del dolor en relación con el temor». La parte práctica, que se realizaba después de la teórica, estaba completamente a cargo de la matrona y en ella se incluían las técnicas respiratorias y de relajación, excluyendo los ejercicios físicos. ${ }^{88}$

\footnotetext{
${ }^{85}$ La dilatación es el periodo del parto durante el que el orificio del cuello del útero se abre hasta posibilitar la salida del bebé. Una vez que se produce la dilatación completa, comienza el periodo expulsivo, en el que el bebé desciende por el canal del parto y que acaba con el nacimiento. El alumbramiento es la salida de la placenta y las membranas (bolsa de las aguas). A veces, la soledad no era lo peor. En las primitivas salas de dilatación las mujeres apenas estaban separadas unas de otras por una cortinilla y el miedo y los gritos de dolor podían «contagiarse» al resto de parturientas.

${ }^{86}$ No obstante, aún existían reminiscencias de la época pasada y los métodos que se recomendaban eran, con preferencia, los denominados «naturales», es decir, los basados en la abstinencia sexual en los días fértiles de la mujer.

87 Yolanda Goyanes Carro, Blanca Bravo López y Mercedes Vázquez García, «La matrona y la educación maternal hospitalaria», en VI Congreso Nacional de Matronas (Palma de Mallorca, [Literatura gris], 1978), 37: Fuente: Literatura gris mecanografiada por la casa Nestlé, colección particular de la autora. Esta afirmación indicaba la necesidad que sentían algunas matronas por ampliar sus horizontes dentro del monótono sistema de atención al parto hospitalario.

88 Blanca Bravo López, Yolanda Goyanes Carro y Mercedes Vázquez García, «El método continuo en la educación maternal», en VI Congreso Nacional de Matronas (Palma de Mallorca, [literatura gris], 1978), 38. Fuente: Literatura gris mecanografiada por la casa Nestlé, colección particular de la autora.
} 
A partir de 1977, el servicio dio un paso más con la convocatoria de cursos de «Educación Maternal para Formación de Monitoras Comarcales». Al menos se celebraron cuatro de estos cursos, el último de ellos destinado a matronas y enfermeras del ámbito rural. ${ }^{89}$

La Residencia Sanitaria «La Paz» también contaba con una sección específica de educación maternal. Aguirre de Cárcer había conseguido, al parecer por nepotismo, la jefatura del servicio. Su puesto le ayudó a monopolizar «buena parte del mercado madrileño de preparación al parto». ${ }^{90} \mathrm{El}$ resto del equipo estaba formado por tres médicos adjuntos, una matrona y una auxiliar de clínica. Esta proporción indica que, todavía en estos momentos, el peso de la actividad recaía más en los profesionales médicos que en las matronas, que fundamentalmente se encargarían de la parte práctica de la preparación.

Además de los cursos organizados en el propio centro hospitalario, las gestantes del área de influencia del centro, que vivían más lejos, podían beneficiarse de la educación maternal en los ambulatorios «Hermanos Aznar»y «Hermanos García Noblejas». Sin embargo, estos centros, que no se habían diseñado para otras actividades que no fuesen las consultas extrahospitalarias y la realización de pruebas complementarias, carecían del espacio suficiente para poder desarrollar la preparación completa. En el «Hermanos García Noblejas», las gestantes tenían que conformarse con los contenidos teóricos debido a la falta de espacio para la gimnasia prenatal. Todos los centros tenían más solicitudes que las que era posible atender y algunas mujeres no pudieron asistir a los cursos. ${ }^{91}$ A pesar de estas deficiencias, en los cinco años transcurridos entre 1975 y 1980 un total de 33.982 mujeres siguieron los cursos.

Esta división del trabajo en el que el tocólogo impartía los conocimientos teóricos, mientras que la parte práctica quedaba en manos de la matrona, era una reproducción de lo que había venido sucediendo en las escuelas de formación para matronas.

89 «I Curso de Educación Maternal para formación de Monitoras Comarcales», 24 al 28 de octubre de 1977, AHC/659321, Biblioteca Nacional de España; «II Curso de Educación Maternal para Formación de Monitoras Comarcales», 6 al 10 de noviembre de 1978, AHC/632891, Biblioteca Nacional de España; «IV Curso de Educación Maternal para formación de Monitoras Comarcales. Destinado a matronas y A.T.S que trabajan en el medio rural», 9, 10 y 11 de diciembre de 1982, AHC/615752, Biblioteca Nacional de España.

${ }^{90}$ Arregui, «Psicoprofilaxis y educación maternal», 118.

91 José Antonio Usandizaga y Francisco Calero (dir.), Ciudad Sanitaria de la Seguridad Social «La Paz» Madrid. Maternidad. Memoria de actividades 1976-1980 (Madrid: Fondo de Investigaciones Sa- 
La sección de Aguirre de Cárcer prestaba su colaboración para establecer otros centros similares en distintas provincias y desarrolló una intensa labor de investigación, acudiendo a diferentes reuniones científicas. Muchas matronas se formaron en la escuela de Aguirre de Cárcer y otras optaron por los cursos impartidos por la Sociedad Española de Sofrología Médica o por la escuela de la noesiterapia de Ángel Escudero. ${ }^{92}$

En 1981, el único centro de Barcelona donde se impartía la educación maternal de manera gratuita era el Hospital Clínico que, como los centros mencionados anteriormente, contaba con su propia Unidad de Educación Maternal. Los cursos ofrecidos por la unidad constaban de once clases teórico-prácticas y se animaba a las mujeres a que fuesen acompañadas por sus parejas. En este caso, el peso de la dirección de la unidad no recaía en un médico sino en la matrona y fisioterapeuta Gloria Sebastiá. Entre sus objetivos estaba «el que la mujer sea realmente la principal protagonista de su maternidad». ${ }^{93}$

Las matronas que se encargaron de abrir el servicio de «Preparación al parto» en el Hospital La Fe de Valencia se formaron previamente en la Escuela Española de Educación Maternal privada de Aguirre de Cárcer en Madrid. ${ }^{94}$

Desde los años cincuenta, algunas matronas se habían percatado de la importancia de asumir el mayor peso de la educación maternal. Esta

nitarias de la Seguridad Social, 1984), 143-144.

92 Ángel Escudero, como se ha visto con anterioridad, había presentado su método en el V Congreso Nacional de Matronas, siendo uno de los conferenciantes invitados. Carmen Garci, una matrona de Castellón de la Plana y practicante del método de Escudero, lo volvió a publicitar en el VI Congreso Nacional de Matronas, asegurando que el efecto anestésico podía durar hasta el octavo día del puerperio y tras una sola relajación: «Intervención de Carmen Garci el 5 de octubre de 1978», en VI Congreso Nacional de Matronas (Palma de Mallorca, 1978), 63. [Literatura gris, mecanografiada por la casa Nestlé, colección particular]. Sobre este método se puede consultar: Ángel Escudero, Curación por el pensamiento (noesiterapia) (Valencia: autoedición, 1998).

${ }_{93}$ J. M. Míguez, «De la maternidad vigilada a la maternidad vigilante. Cómo son y por qué son necesarios los cursillos de preparación al parto», La Vanguardia, 1 de agosto de 1981: 34. Gloria Sebastiá, como hemos visto con anterioridad, también era la presidenta de la Sección Española de la Federación Mundial de Sofrología.

${ }_{94}$ Para conocer los detalles pormenorizados de cómo se realizaban estos cursos y lo que supuso para las matronas asumir poco a poco esta competencia puede consultarse M. ${ }^{\mathrm{a}}$ José Alemany Anchel, De mujeres y partos. Matronas y cambio social en la segunda mitad del siglo XX (València: Universitat de València, 2016): 192-196. 
función docente podía llenar el hueco que habían dejado los partos domiciliarios y que había vaciado de contenido la presencia de las matronas contratadas por los ayuntamientos. Además, era una forma de recuperar una parte de la autonomía perdida que había supuesto la institucionalización del parto. A pesar de todo, las cosas no iban a ser tan sencillas como parecía.

Cuando empecé yo en el 86 a hacer preparación a la maternidad en [el pueblo] pues desde [el hospital de referencia] el doctor [el jefe de servicio] se rasgó las vestiduras y dijo que quien era una matrona para hacer eso y pidió referencias e informes y a ver. Que dónde estaba, le presentaron mi programa de cómo iba a hacerlo todo, y que dónde estaba, que sí que sí que todo muy bien, pero dónde estaba el médico responsable de eso. Por aquel entonces no existían protocolos, no existía nada. Yo cuando llegué a [el pueblo] no había Atención Primaria. Mi despacho al principio era el coche. ${ }^{95}$

Pero no todas las matronas estaban dispuestas a aceptar nuevos retos tras muchos años de desarrollo profesional. La situación iba a cambiar, diametralmente, con la implantación de la Atención Primaria y en dicho proceso resultó fundamental la matrona Rosario Muñoz González, que actuó como asesora del Ministerio de Sanidad y de la Dirección General de Atención Primaria. ${ }^{96}$ El objetivo inicial de Rosario Muñoz y su grupo era conseguir que las matronas se incorporasen como miembros de pleno derecho a los equipos de Atención Primaria. Sin embargo, y debido a diversos obstáculos, ${ }^{97}$ la incorporación se produjo como miembros de unidades de apoyo, es decir, personal no imprescindible y ajeno al equipo, con la consiguiente imposibilidad de participar en la toma de decisiones de este. Rosario Muñoz participó en la elaboración, en 1984, de un proyecto específico de la reforma de la figura de la matrona, que iba a tener dos actividades fundamentales: la educación para la salud a grupos

\footnotetext{
95 Entrevista a H.G.P. realizada el 10 de octubre de 2018.

96 Entrevista telefónica a Rosario Muñoz González, el 7 de noviembre de 2020.

97 Por ejemplo, la oposición de Aguirre de Cárcer, que se reunió con el subdirector de Atención Primaria de la Seguridad Social «con la intención de evitar la previsible chapuza»: García Arregui, «Psicoprofilaxis y educación maternal», 123.
} 
y las consultas de matronas. ${ }^{98}$ Estas actividades formaban parte del Programa Integral de Atención a la Mujer y suponían desarrollar las competencias autónomas que habían sido reconocidas ya por la Comunidad Económica Europea. ${ }^{99}$ Estas profesionales iban a ser las responsables de la puesta en marcha de las Unidades de Psicoprofilaxis Obstétrica en los Centros de Atención Primaria. Para ello, se organizaron diversos cursos de formación para matronas que fueron impartidos por Gloria Sebastiá, la cual se desplazaba a Madrid desde Barcelona. ${ }^{100}$ La nueva modalidad de matrona de Atención Primaria se concretó en una Resolución del Ministerio de Sanidad y Consumo de 19 de febrero de 1990. ${ }^{101}$

\section{CONCLUSIONES}

Aunque se incorporaron tardíamente a los foros de toma de decisiones, las matronas tuvieron un papel crucial en la implantación y la difusión de la educación maternal en España, especialmente en las zonas rurales y dentro del sistema público.

Las matronas comprendieron que su universo laboral, que siempre había girado en torno al parto domiciliario, se estaba transformando irremediablemente con el advenimiento de los seguros sociales y la generalización del parto hospitalario. Asumir competencias en la educación maternal era una forma de ampliar su horizonte, paulatinamente constreñido por los médicos en los siglos anteriores. Para muchas matronas, la educación maternal fue la excusa perfecta para huir de unas instituciones cerradas en las que la jerarquía, todavía hoy en día, limita su autonomía profesional, reconocida internacionalmente por entidades como la Organización Mundial de la Salud o las Naciones Unidas.

\footnotetext{
98 Instituto Nacional de la Salud. Subdirección General de Gestión de Atención Primaria, Introducción al trabajo de la matrona en atención primaria (Madrid: INSALUD, Secretaría General, Servicio de Publicaciones, 1987).

99 Rosario Muñoz González, «Nueva modalidad de matrona en Atención Primaria», Matronas Hoy, n. 2 (1992): 16-17.

100 Entrevista telefónica a Rosario Muñoz González, el 7 de noviembre de 2020.

101 Resolución de 19 de febrero de 1990, de la Subsecretaría, por la que se ordena la publicación del acuerdo del consejo de Ministros, por el que se amplía el Real Decreto-ley 3/2987, a determinada categorías de personal que presta servicios en el INSALUD.
} 
Independientemente de que Consuelo Ruiz Vélez-Frías perdiese la batalla contra Aguirre de Cárcer por compartir protagonismo en la implantación de la educación maternal en España, no se puede afirmar que existiera «una ausencia generalizada de este colectivo en la promoción del parto si dolor».102

Por último, una reflexión: ¿Tienen sentido los cursos colectivos para embarazadas hoy en día? En la actualidad la mortalidad infantil es mínima y disponemos de diversos métodos para calmar el dolor en el parto, entre ellos la anestesia epidural, cuyo uso se generalizó en 1999 para todas las mujeres que la deseasen. ${ }^{103}$ Tampoco es un problema el acceso a la información, cuyo volumen en las redes es prácticamente inabarcable. A esto hay que sumar los libros y revistas sobre salud materno-infantil, que siguen siendo un valor editorial seguro en estos tiempos inciertos. $\mathrm{Y}$, sin embargo, las mujeres y sus parejas siguen demandando inscribirse en las clases pre y postnatales de los centros de atención primaria. Probablemente porque la individualidad a la que nos aboca la sociedad occidental pierde sentido cuando un nuevo ser se está gestando: «para educar a un niño/a, hace falta toda una tribu», o mejor, como señala José Antonio Marina, ${ }^{104}$ no cualquier tribu sino una buena tribu. Los grupos de parejas gestantes se convierten, en ocasiones, en esa tribu, y las clases suponen una parte del rito de transición de convertirse en madres y padres.

Durante el confinamiento general y el proceso de desescalada que sufrió España, entre el 13 de marzo y el 21 de junio de 2020, para evitar la expansión del virus SARS-CoV-2, las clases grupales para embarazadas se cancelaron. A falta de medios apropiados, o de directrices claras de actuación, por parte de unos equipos de gestión sobrepasados por la emergencia sanitaria, muchas matronas improvisaron métodos alternativos para suplir la falta de presencialidad. Los vídeos tutoriales y las reuniones virtuales a través de redes sociales y plataformas informáticas fueron algunos de los recursos utilizados, y parece que han llegado para quedarse en mayor o menor medida.

102 García Arregui, «Psicoprofilaxis y educación maternal», 45.

${ }^{103}$ A excepción de los casos en que existiese una contraindicación médica.

104 José Antonio Marina Torres, «¿Qué es la movilización educativa?», Cuadernos de pedagogía, n. 367 (2007): 57-59. 
Muchas matronas actuales rechazan usar el concepto de «educación maternal», aunque es el que aparece en la cartera de servicios, y prefieren utilizar el de "preparación para el nacimiento y la crianza», que les parece más inclusivo y holístico. Tenga el nombre y la forma que tenga, parece que estos espacios de encuentro entre gestantes y matronas no van a desaparecer, ni siquiera con una pandemia de por medio.

\section{Nota sobre la autora}

Dolores Ruiz-BERdún es doctora por la Universidad de Alcalá (2012). Profesora titular de Historia de la Ciencia en la Universidad de Alcalá. Secretaria de la Sociedad Española de Historia de las Ciencias y de las Técnicas (SEHCYT). Sus líneas de investigación se centran en los aspectos históricos y antropológicos de la atención al parto y de sus profesionales, la historia del sistema sanitario español, la historia de la educación sanitaria (siglos XIX-Xx) y la historia de la salud materno-infantil (siglos XVIII -Xx). Ha participado y participa en diversos proyectos de investigación nacionales e internacionales. Ha sido galardonada con el premio «María Isidra de Guzmán» destinado a estudios de género por el libro Compromiso Social y Género. La historia de las matronas en la II República, la Guerra Civil y la autarquía (1931-1955) (edición 2016), con el premio «Cátedra Antonio Chamorro-Alejandro Otero» otorgado por la Real Academia de Medicina y cirugía de Andalucía Oriental por el artículo «Matronas víctimas de la Guerra Civil española» (Asclepio, 2016), ambos trabajos en coautoria con Alberto Gomis y en solitario con el premio «Maribel Bayón» edición 2019 por el artículo «Matronas, pioneras en la universidad desde 1845» (Matronas Hoy, 2020). Entre sus publicaciones más recientes se encuentran el artículo «Being a woman, being a soldier, being a mother: a qualitative analysis of perceptions of pregnancy on working lives of women in the Spanish Armed Forces» (British Medical Journal Military Health, 2021) en coautoria con Sheima Hossain-López, y el capítulo «Las dificultades de las matronas en la España rural del siglo xx» en el libro editado por José Martínez Pérez y Enrique Perdiguero Gil: Genealogías de la reforma sanitaria en España (La Catarata, 2020). 


\section{REFERENCIAS}

Alemany Anchel, M. ${ }^{a}$ José. De mujeres y partos. Matronas y cambio social en la segunda mitad del siglo xx. València: Universitat de València, 2016.

Ballarín Domingo, Pilar. "La educación de la mujer española en el siglo XIX». Historia de la Educación. Revista interuniversitaria 8 (1989): 245-260.

Bernabeu Mestre, Josep y Encarna Gascón Pérez. «El papel de la enfermería en el desarrollo de la salud pública española (1923-1935): la visitadora sanitaria», Dynamis 15 (1995): 151-176.

Colmenar Orzaes, Carmen. «La institucionalización de la maternología en España durante la Segunda República y el franquismo». Historia de la Educación, n. ${ }^{\circ} 28$ (2009): 161-183.

Donnison, Jean. Midwives and medical men. A History of Interprofessional Rivalries and Women's Rights. London: Heinemann, 1977.

Escudero, Ángel. Curación por el pensamiento (noesiterapia). Valencia: autoedición, 1998.

Fernández y Fernández-Arroyo, Matilde. «Elaboración, validación y aplicación de una herramienta de evaluación para los programas de Educación Maternal, de los Centros de Atención Primaria de la Comunidad de Madrid». PhD diss., Universidad Pontificia de Comillas de Madrid, 2012.

Fernández y Fernández-Arroyo, Matilde. «Análisis comparativo de las principales Escuelas de Educación Maternal». Index de Enfermería 22, n. ${ }^{\circ}$ 1-2 (2013): 40-44.

García Arregui, Amaya. "Psicoprofilaxis y educación maternal. Historia de la preparación al parto en España». PhD diss., Universidad Autónoma de Barcelona, 2019.

González de Pablos, Ángel. «Las nuevas ciencias del espíritu del siglo xx: La aparición de la sofrología en España a inicios del segundo franquismo». Cultura Psi, n. ${ }^{\circ}$ (2016): 91-117.

González Pérez, Teresa. «El aprendizaje de la maternidad: discursos para la educación de las mujeres en España». Convergencia. Revista de Ciencias Sociales, n. ${ }^{\circ} 46$ (2008): 91-117.

González Pérez, Teresa. «Los programas escolares y la transmisión de roles en el franquismo: la educación para la maternidad», Bordón 61, 3 (2009): 93-105.

Gozalbo Gimeno, Daniel. «Historia archivística de los expedientes de censura editorial (1942-2017)». Creneida 5 (2017): 8-34.

Jiménez Lucena, Isabel, María José Ruiz Somavilla y Jesús Castellanos Guerrero. «Un discurso sanitario para un proyecto político. La educación sanitaria en los medios de comunicación de masas durante el primer franquismo», Asclepio 54, n. ${ }^{\circ} 1$ (2002): 201-218. 
Marina Torres, José Antonio. «¿Qué es la movilización educativa?», Cuadernos de pedagogía n. 367 (2007): 57-59.

Martín-Alcaide, Rosario y Ruiz-Berdún, Dolores. «Un hospital para mujeres promovido por mujeres: la Casa de Salud de Santa Cristina de Madrid». Temperamentvm 15 (2019), e12642 http://ciberindex.com/index.php/t/article/view/e12642

Ortiz Gómez, Teresa. «De matrona a matrona. Francisca Iracheta y la divulgación de la ciencia obstétrica en España en 1870». Arenal 6, n. ${ }^{1}$ (1999): 183-195.

Palacio Lis, Irene. Mujeres ignorantes: madres culpables. Adoctrinamiento y divulgación materno-infantil en la primera mitad del siglo xx. València: Universitar de València, 2003.

Palacio Lis, Irene. "Consejos a las madres": autoridad, ciencia e ideología en la construcción social de la función materna. Una mirada al pasado». Sarmiento, n.o 7 (2003): 61-79.

Perdiguero Gil, Enrique (comp.). Salvad al niño. Valencia: Seminari de Estudis sobre Ciència, 2004.

Perdiguero Gil, Enrique, y Mercedes Del Cura. «Introducción». En Salvad al niño, compilado por Enrique Perdiguero Gil, 15-25. Valencia: Seminari de Estudis sobre Ciència, 2004.

Pons Pons, Jerònia y Margarita Vilar Rodríguez. El seguro de salud privado y público en España. Su análisis en perspectiva histórica. Zaragoza: Prensas de la Universidad de Zaragoza, 2014.

Ponte Hernando, Fernando et al. «Tal como éramos. En el centenario del primer Congreso Español de Pediatría de Palma de Mallorca (1914-2014)». Anales de Pediatría 80, n. ${ }^{\circ}$ 6. doi: 10.1016/j.anpedi.2014.03.010

Rodríguez Ocaña, Esteban. «Aspectos sociales de la pediatría española anteriores a la Guerra Civil (1936-39)». En La ciencia moderna y el conocimiento del Nuevo Mundo: actas de la I Reunión de Historia de la Ciencia y de la Técnica de los Países Ibéricos e Iberoamericanos, editado por José Luis Peset Reig, 443460. Madrid: Consejo Superior de Investigaciones Científicas, 1985.

Rodríguez Ocaña, Esteban. «La construcción de la salud infantil. Ciencia, medicina y educación en España». Historia Contemporánea, n. 18 (1998): 19-52.

Rodríguez Ocaña, Esteban y Enrique Perdiguero. «Ciencia y persuasión social en la medicalización de la infancia en España, siglos XIX-XX». História, Ciências, Saúde-Manguinhos 13, n. ${ }^{\circ}$ 2, (2006): 303-324. doi: https://doi.org/10.1590/ S0104-59702006000200007

Ruiz-Berdún, Dolores. «Pilar Primo de Rivera y la reorganización de las carreras auxiliares sanitarias tras la Guerra Civil», en La tutela imperfecta. Biología y Farmacia en la España del primer franquismo, editado por Antonio González Bueno y Alfredo Baratas Díaz, 81-100. Madrid: Consejo Superior de Investigaciones Científicas, 2013. 
Ruiz-Berdún, Dolores. «Evolución de la fabricación, comercialización y uso de los derivados del cornezuelo de centeno en Obstetricia». En De la botica de El Escorial a la industria farmacéutica. En torno al medicamento editado por Alberto Gomis Blanco y Raúl Rodríguez Nozal, 333-359. Alcalá de Henares: Servicio de Publicaciones de la Universidad de Alcalá, 2015.

Ruiz-Berdún, Dolores. «Las dificultades de las matronas en la España rural del siglo xx». En Genealogías de la Reforma Sanitaria en España, editado por José Martínez Pérez y Enrique Perdiguero Gil, 21-62. Madrid: Los Libros de la Catarata, 2020.

Ruiz Vélez-Frías, Consuelo. «La sabiduría silenciada». En Hilando fino. Mujeres, un viaje en común, 233-277. Barcelona: Icaria, 2007.

Sage Pranchère, Nathalie. "La résistance d'une profession», en L'école des sages femmes: Naissance d'un corps professionel, 1786-1917 (Tours: OpenEdition Books, 2017). doi: https://doi.org/10.4000/books.pufr.13223.

Salazar Agulló, Modesta, Josep Bernabeu-Mestre, Encarnación Ramos Salas y María Eugenia Galiana Sánchez. "Madres y enfermeras: el modelo de cuidados a la infancia en la colección "Al servicio de España y del niño español" (1938-1964)». Recien. Revista científica de Enfermería 1 (2010). http://hdl.handle.net/10045/20315

Torres Díaz, Ángela. «Un manual para el adoctrinamiento de las matronas», Temperamentvm 13 (2017). https://ciberindex.com/index.php/t/article/view/e11600.

Villa Rodríguez, Luciano de la. «Presentación». En Escuela Nacional de Puericultura 1926-1976. Cincuenta aniversario de la actividad docente y asistencial. (Madrid: Ministerio de Sanidad y Seguridad Social, Subsecretaría de la Salud, 1978).

Viñao, Antonio. «La educación en las obras de Josefa Amar y Borbón». Sarmiento, n. 7 (2003): 35-60.

Vuille, Marilène. «L'Obstétrique sous influence: émergence de l'accouchement sans douleur en France et en Suisse dans les années 1950», Revue d'histoire moderne \& contemporaine 64, n. 1 (2017): 116-149. 